\title{
Rheological model of dynamic pupillary reflex in the assessment of the level of autonomic nervous system activation
}

\author{
K. Różanowski, O. Truszczyński, J. Lewandowski \& M. Madeyski \\ Aviation Bioengineering Department, \\ Military Institute of Aviation Medicine, Poland
}

\begin{abstract}
The aim of this publication was to adapt a rheological model of Kelvin-Voigt in the description of the dynamic pupillary reflex. The pupillary reflex presents the results of physiological adaptation mechanisms in the human eye, i.e. response of the iris to the changing intensity of light falling on the retina under the condition of sensory deprivation. Pupillary light reflex (PLR) is a closed loop nerve reflex.

The new approach to PLR evaluation presented by the authors consists of adapting the Kelvin-Voigt model to the description of this phenomenon. The authors, for their tests of correct implementation of the model, used data from pilot studies. Research was performed on a group of 10 volunteers in a laboratory environment, at night. The mean age of the study participants was $27 \pm 0.4$ years. For PLR registration, the $\mathrm{F}^{2} \mathrm{D}$ Fit-For-Duty system was used .The system provides useful data for shift work or for personnel in high-risk careers.

The PLR measurements were taken for both eyes, which were exposed to a series of five flashes at 30 -second intervals. The whole measurement of both eyes was conducted with patients wearing goggles, in order to maintain the same measurement conditions, mostly with regard to accommodation.

Owing to proper implementation of the model, we managed to attain a high level of its adaptation to real data $(>90 \%)$. The results show their strong correlation, and the model shows high sensitivity to changes in the shape of the reflex curve, caused by sleep deprivation introduced in the experiment.

Keywords: pupillary light reflex, fatigue, rheological model, Kelvin-Voigt model.
\end{abstract}




\section{Introduction}

Experimental studies associated with the recording and assessment of pupil behavior involved a measurement system featuring optical sensors that constituted an interesting combination of hardware and software solutions [1, 2]. This specific class of optical sensors based on video image analysis is used in pupillometry and facilitates identification of eyeball movements as well as determination of parameters describing the dynamics of eye shutting motion and dynamic pupillary change reaction. The evaluation of pupil motion provides functional information about the autonomous nervous system [3-5]. Pupillary light reflex (PLR) is the response of the iris to changing intensity of incident light at the retina. The reflex is modulated by eye accommodation status as well as factors of sensory and emotional origin. The effectors consist in constricting and relaxing muscles of the iris that attempt to maintain a constant level of retinal illumination by changing the size of the iris. Temporal characteristics of PLR depends on the status of two antagonistic systems of the autonomous nervous system $(A U N)$, i.e. sympathetic nervous system $(S N S)$ and parasympathetic nervous system (PNS).

The PLR has already been studied to detect a $P N S$ defect in various affections: alcoholism, diabetes, AIDS, depression, anxiety, drug addiction, schizophrenia. Since pupillary dilation is regulated autonomically, an effort was made to use this method as a valid measure of pain [6-10]. Research using pupillometry is being carried out to assess alertness of hypersomnolent patients. Observation of pupil behavior is also used on clinically sleepy patients, as well as in the assessment of cardiac autonomic function of sport athletes [11]. As shown, this method has a variety of clinical applications. Furthermore, the identification of pupil dynamics is also the subject of research concerning the monitoring of various areas of human behavior [12].

\section{Materials and methods}

\subsection{Implementation of the model}

An $\mathrm{F}^{2} \mathrm{D}$ Fit-For-Duty, a commercial system by AMTech, was used to record the PLR. The system provides useful data for shift work or for personnel in high-risk careers. Areas of application are occupational medicine, sleep medicine and pharmacology. The system is a non-invasive tool to obtain an objective assessment of the central nervous activation and drug-related vigilance. There are several predefined parameters that may be used to describe PLR. Parameters determined using the $\mathrm{F}^{2} \mathrm{D}$ Fit-For-Duty device by AMTech are as follows: latency $L$ describes the delay between a light pulse and the pupil reaction; duration of reaction $D R$ is the time to reach minimal value diameter; time of minimum diameter $T M D$ is the time for which diameter is less than $50 \%$ of the beginning value; $2 / 3$ contraction interval $2 / 3 C I$ is the time in which the amplitude reaches $1 / 3$ of the value in contraction phase; $1 / 3$ redilatation interval $1 / 3 R I$ represents the time in which the amplitude reaches $1 / 3$ of the value in 
redilatation phase; and amplitude $A$ describes the difference between the maximum and minimum value of the diameter [13-15].

However, the parameters account for the dynamic character of pupillary changes only at strictly defined time points. The authors propose an implementation of the Kelvin-Voigt's model to assess the changes in pupillary behavior and the effect of ANS on PLR that would facilitate identification of the dynamic character of PLR over the entire duration of the reflex, not just in selected measurement points and parameters associated thereof.

The size of the human pupil (diameter or area) is determined by antagonistic activity of the muscles of the iris, i.e. pupillary constrictor and dilator muscles, innervated by the parasympathetic and sympathetic system, respectively. Pupillary constrictor consists of smooth muscle fibers and has the shape of a flattened ring, $1 \mathrm{~mm}$ wide, located within the posterior part of the stroma of the iris, close to the pupillary edge. Pupillary dilator consists of radially arranged muscle fibers spanning from the periphery of the iris towards the center of the pupil. The mechanism of action of these muscles is illustrated schematically in Fig. 1.

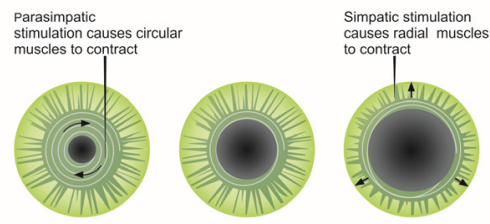

Figure 1: Schematic illustration of the mechanism of the iris response to a light stimulus.

PLR is a closed neural feedback loop system. The purpose of the reflex is to regulate the amount of light incident on the retina (by means of changing the pupil size). A schematic diagram of the PLR reflex arc is presented in Fig. 2.

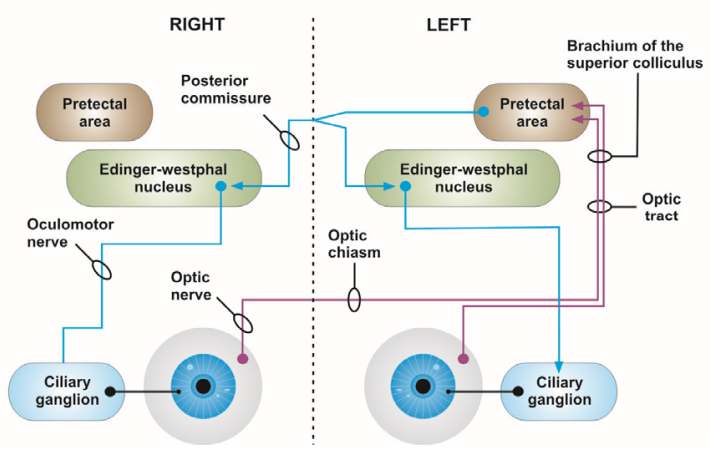

Figure 2: Schematic diagram of the reflex arc for the pupillary light reflex. 
The dynamics of the pupillary light reflex may be described by means of rheological models that describe the behavior of bodies subjected to strains which cannot be described by means of ideally elastic body models. Deformation creep, i.e. an increase in deformation caused by constant normal strain exerted on a body, is an important phenomenon in such cases. Creep may be described by means of the Kelvin-Voigt's model (illustrated in Fig. 3), and mathematical formula:

$$
\varepsilon(t)=\frac{\sigma_{0}}{E}\left(1-e^{\frac{-E}{\eta} t}\right)
$$

where: $\varepsilon(t)$ - total deformation, $\sigma_{0}$ - initial deformation, $E$ - modulus of longitudinal elasticity, $\eta$-dynamic viscosity, $t$ - time.

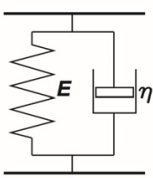

(a)

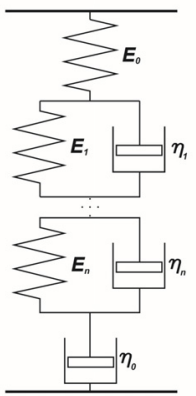

(b)

Figure 3: Diagrams of the Kelvin-Voigt model: (a) schematic; (b) general.

A bi-element model is too simple to describe actual biological materials. Therefore, subsequent elements are added to the model until a satisfactory model accuracy is achieved. A generalized model is presented schematically in Fig. 3(b) and its mathematical representation is described by equation (2).

$$
\varepsilon(t)=\sigma_{0}\left(\frac{1}{E_{0}}+\sum_{i=1}^{n} \frac{1}{E_{i}}\left(1-e^{\frac{-i}{\tau_{i}}}\right)+\frac{1}{\eta_{0}}\right)
$$

where: $\tau_{i}$ - relaxation time.

Since the two iris muscles play essential roles in controlling the pupil size, their dynamic properties must be considered in the PLR model. The constrictor and dilator muscles could be modeled as viscoelastic materials using the KelvinVoigt model in a circular and linear shape, respectively. The movement of the iris muscles is governed by three forces: passive muscle elastic force, viscous resistance, and the active forces generated from the ANS modulation. The constrictor is modulated by the parasympathetic system, whereas the dilator is modulated by the sympathetic system. Let the radius of constrictor be $r c$, and the length of dilator muscle be $r d$. Assuming the constrictor has a circular shape with a perimeter of $2 \pi r_{c}$, the dynamic equations can be constructed for constrictor and dilator as: 


$$
\begin{aligned}
& m_{c} \frac{d^{2} r_{c}}{d t^{2}}=-2 \pi P_{e c}+P_{v c}-\frac{F_{p}(t)}{2 \pi} \\
& m_{d} \frac{d^{2} r_{d}}{d t^{2}}=-P_{e d}+P_{v d}-F_{s}(t)
\end{aligned}
$$

where: $m_{c}$ - the mass for constrictor (in $\mathrm{mg}$ ), $m_{d}$ - the mass for dilator (in $\mathrm{mg}$ ), $P_{e c}$ - the viscous resistance in the constrictor, $P_{e d}-$ the viscous resistance in the dilator.

The passive iris muscle elasticity was modeled as a second-order equation of muscle length based on experimental observation [16]:

$$
\begin{gathered}
P_{e c}= \begin{cases}k_{c}\left(\mathrm{r}_{\mathrm{c}}-l_{0 \mathrm{c}}\right)^{2}, & \left(\mathrm{r}_{\mathrm{c}} \geq l_{0 \mathrm{c}}\right) \\
0, & \left(\mathrm{r}_{\mathrm{c}}<l_{0 \mathrm{c}}\right)\end{cases} \\
P_{e d}= \begin{cases}k_{d}\left(\mathrm{r}_{\mathrm{d}}-l_{0 \mathrm{~d}}\right)^{2}, & \left(\mathrm{r}_{\mathrm{d}} \geq l_{0 \mathrm{~d}}\right) \\
0, & \left(\mathrm{r}_{\mathrm{d}}<l_{0 \mathrm{~d}}\right)\end{cases}
\end{gathered}
$$

where: $k_{c}, k_{d}$ - elastic constants, $l_{0 c}-$ the radius of the constrictor at rest, $l_{0 d}-$ the dilator muscle resting length.

$P_{v c}$ and $P_{v d}$ were modeled as a linear function of velocity [17]:

$$
\begin{gathered}
P_{v c}=-D_{c} \frac{d r_{c}}{d t} \\
P_{v d}=-D_{d} \frac{d r_{d}}{d t}
\end{gathered}
$$

where: $D_{c}, D_{d}$ - viscous constants.

The $F p(t)$ in (9) and $F s(t)$ in (10) are the muscle forces originated from the parasympathetic and sympathetic system induced by a light stimulus, respectively. These are effective forces that could be affected by both the afferent and efferent pupillary pathway. For a short-pulse stimulus, the forces originated from the parasympathetic and sympathetic system were assumed to be square-wave pulses with different delays, $\tau_{p}$ and $\tau_{s}$ and durations, $\Delta t_{p}$ and $\Delta t_{s}[18]$.

$$
\begin{aligned}
& F_{p}(t)= \begin{cases}f_{p}+f_{p 0}, & \left(\tau_{\mathrm{p}} \leq t \leq \tau_{p}+\Delta t_{p}\right) \\
f_{p 0}, & \left(t<\tau_{\mathrm{p}}, t>\tau_{p}+\Delta t_{p}\right)\end{cases} \\
& F_{s}(t)= \begin{cases}f_{s}+f_{s 0}, & \left(\tau_{\mathrm{s}} \leq t \leq \tau_{s}+\Delta t_{s}\right) \\
f_{s 0}, & \left(t<\tau_{\mathrm{s}}, t>\tau_{s}+\Delta t_{s}\right)\end{cases}
\end{aligned}
$$

where: $f_{p 0}, f_{s 0}$ - the muscle forces originated from the parasympathetic and sympathetic systems at the resting condition.

\subsection{Implementation of the model}

Data obtained from the measurements of the pupillary reflex were submitted to preprocessing, consisting in data filtration using a "Savitzky-Golay" filter with polynomial order of 3 and data length of 7 . Filter parameters were selected empirically. Filtration resulted in the smoothing of the acquired data. Next, 
spline interpolation was carried out to increase the number of measurement points from 51 to 201. As a result, the sampling frequency was artificially increased from 25 to $100 \mathrm{~Hz}$.

Preliminary data processing significantly reduced the root mean square percentage error (RMSPE) calculated from the following formula:

$$
R M S P E=100 * \sqrt{\frac{1}{N} \sum_{i=1}^{N}\left(\frac{x_{i}-y_{i}}{x_{i}}\right)^{2}}
$$

The parameters were estimated at possibly lowest RMSPE values for each experiment.

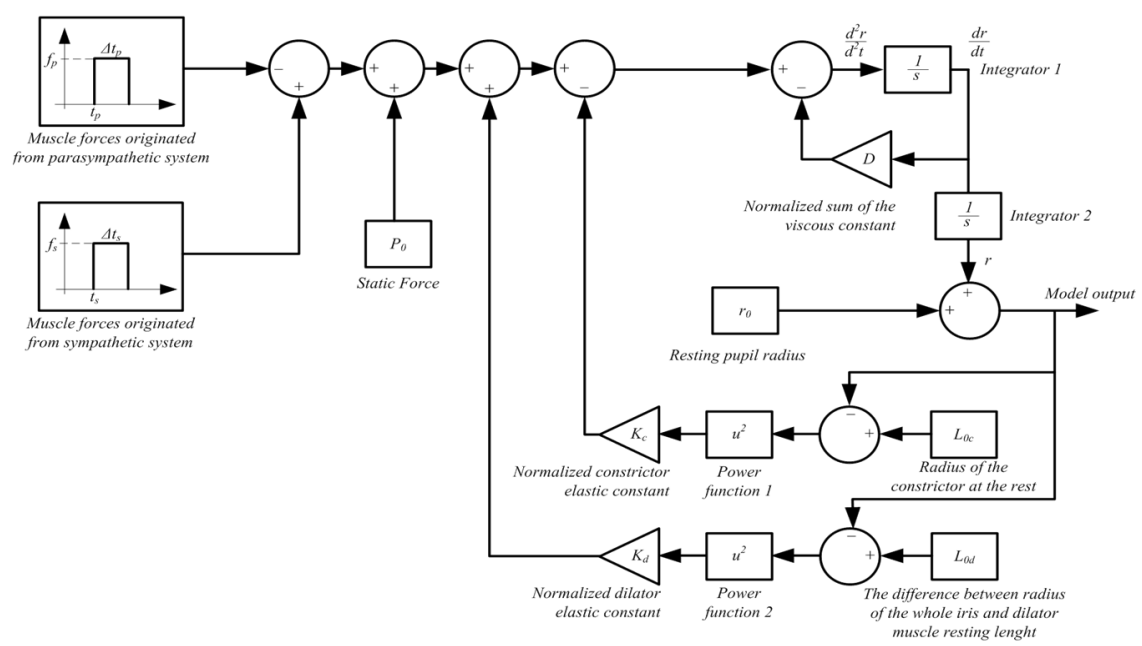

Figure 4: Block diagram of the implementation of the mathematical model of pupillary reflex triggered by a short-lived light pulse.

Fig. 4 presents a block diagram of the implementation of the mathematical Kelvin-Voigt's model for the assessment of pupillary reflex triggered by a shortlived light pulse. The model consists of blocks representing a set of parameters and constraints describing the movement of the iris. The constraints are in the shape of square pulses reflecting activation of sympathetic and parasympathetic nervous systems. As a result of running the model, one obtains a plot of pupillary radius as a function of time following the light pulse. The developed software also facilitates identification of the model parameters (Table 1), while placing any constraint in the form of an actual pupil size signal recorder during the examination. The model was implemented in the Matlab R2014b environment. The parameters of the PLR model are presented in Table 1.

Fig. 5 presents the results obtained from the PLR model when estimating the parameters on the basis of raw and preprocessed data. 
Table 1: List of parameters determined in the dynamic PLR assessment model.

\begin{tabular}{|l|l|l|}
\hline No. & Symbol & Description \\
\hline 1. & $\mathrm{D}$ & Sum of viscous constant \\
\hline 2. & $\mathrm{~K}_{\mathrm{c}}$ & Constrictor elastic constant \\
\hline 3. & $\mathrm{~K}_{\mathrm{d}}$ & Dilator elastic constant \\
\hline 4. & $\mathrm{P}_{0}$ & Resting pupil static force \\
\hline 5. & $\mathrm{~L}_{0 \mathrm{c}}$ & Radius of the constrictor at rest \\
\hline 6. & $\mathrm{~L}_{0 \mathrm{~d}}$ & Pupillary resting radius minus dilator muscle resting length \\
\hline 7. & $\mathrm{r}_{0}$ & Pupillary resting radius \\
\hline 8. & $\mathrm{t}_{\mathrm{p}}$ & Delay force originated from the parasympathetic system \\
\hline 9. & $\mathrm{t}_{\mathrm{s}}$ & Delay force originated from the sympathetic system \\
\hline 10. & $\mathrm{f}_{\mathrm{p}}$ & Value of parasympathetic force \\
\hline 11. & $\mathrm{f}_{\mathrm{s}}$ & Value of sympathetic force \\
\hline
\end{tabular}

(a)

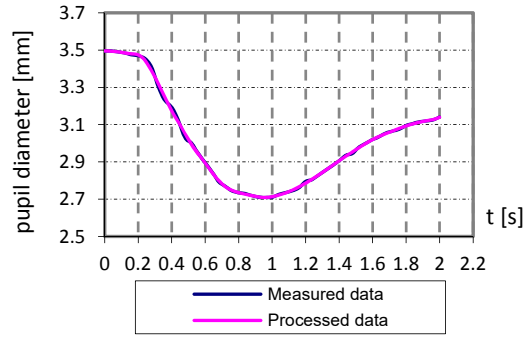

(c)

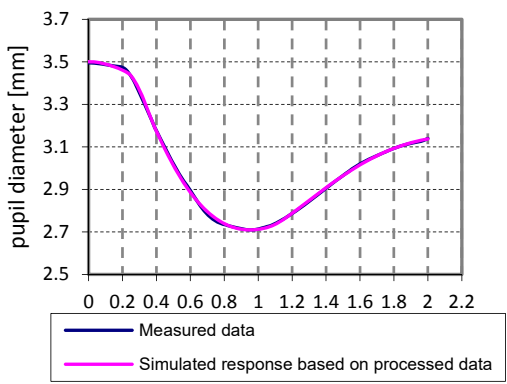

(b)

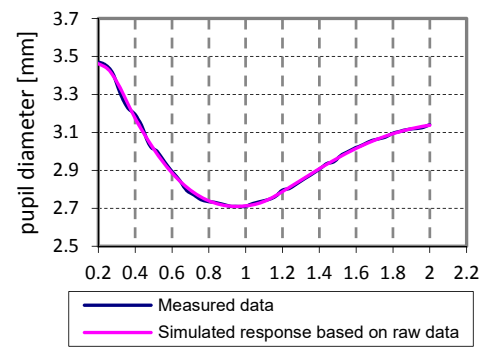

(d)

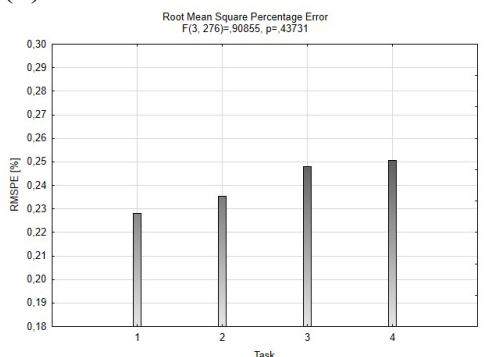

Figure 5: Pupil size signals recorded during the PLR: (a) raw data and preprocessed data; (b) actual data and PLR model-generated data when estimating model parameters from raw data; (c) actual data and PLR model-generated data when estimating model parameters from data after preprocessing for file 33_301_LE_0; (d) histogram of RPMSE values for individual measurements.

As seen in Fig. 5, the model fit is close to $100 \%$, particularly when the model parameters are estimated from preprocessed data. The fit level is independent of 
the measurement, i.e. it closely follows the curve reflecting the pupil size changes occurring as fatigue increases (Fig. 6).

\subsection{Experimental}

\subsubsection{Study subjects}

Research was performed at night on a group of 10 volunteers in a laboratory environment. Their mean age was $27 \pm 0.4$ years. Each study participant was informed about the study design and provided an informed consent [19]. On the day of experiment, participants abstained from caffeine intake of any form for at least 4 hours before the test and for the entire duration of the test. Additionally, in accordance with methodology requirements, each participant spent 7-8 hours resting the night before the study. Each participant performed the test 4 times between $11 \mathrm{pm}$ and 6:30 am.

\subsubsection{Experimental procedure}

The measurement of participants' PLR was one of the elements of the experiment. It also included assessment of subjective level of fatigue using Japanese Questionnaire and the level of alertness measured by SSS (Stanford Sleepiness Scale), which is a quick and easy way to assess a person's level of alertness. Test using the APK apparatus was also performed. The device is intended for psychological tests for i.a. drivers and heavy-machinery operators in terms of the speed of psychomotor reaction at an imposed or random rate, eyehand coordination skills, perception speed and accuracy, time-limited decisionmaking and resistance to fatigue. Simple psychological tests were designed only to control psychomotor ability throughout the study. They did not have an impact on the main objective of the experiment. Measurements utilizing the goggles began with an 11-minute PLR test as required by the procedure, followed by the PLR test at 2-minute intervals. PLR measurement was performed on both eyes which were subjected to a series of 5 flashes at 30 -second intervals. Repeat eyeball stimulation with a source of light is the result of work performed by researchers on the measurement of physiological parameters. The right eye was subjected to light stimuli first. The duration of pupillary reflex measurement was 150 seconds. Then, over a period of 60 seconds, the system switched to the left eye, which similarly to the right eye, was subjected to a series of 5 impulses of light over a period of 150 seconds. The entire process was carried out with the subjects wearing goggles to ensure the same measurement conditions, mainly in terms of accommodation.

\subsubsection{Experimental results}

The implemented model was verified using the experimental data recorded as described in the Experimental procedure section. The estimated parameters, Table 3, were compared to the classical parameters that describe the PLR. Plots of relative amplitude and contraction velocity are presented in the article. Both parameters are characterized by the strongest correlations with the declared subjective fatigue levels as well as with the reference parameter, i.e. the pupillary unrest index (PUI) [20]. As clearly shown in Fig. 6, both parameters change along with the increasing fatigue (sequential measurements Task 1-Task 2). 
(a)

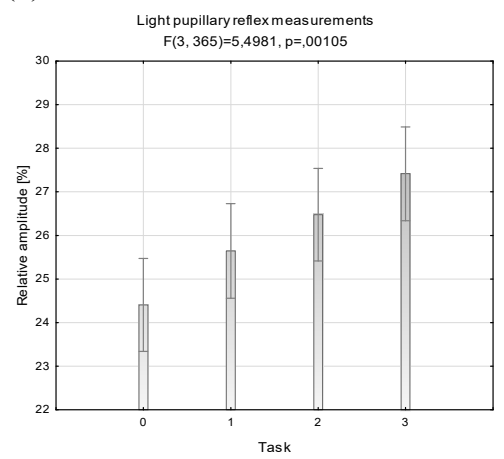

(b)

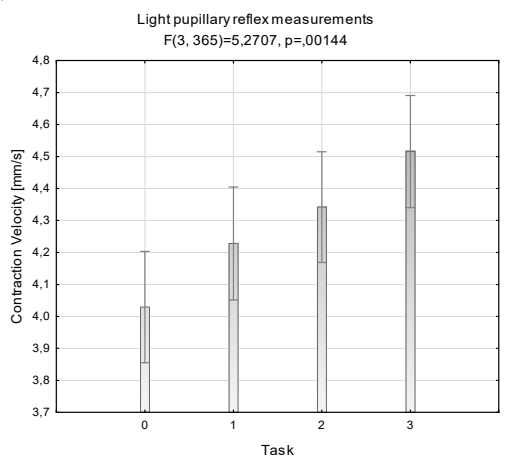

Figure 6: Graph changes: (a) amplitude of contraction; (b) rate of pupillary constriction in subsequent stages of measurement.

The analysis of the $t_{s}, t_{p}, f_{s}, f_{p}$ model parameters revealed variable behaviors in sequential measurements (Task 1-Task 4) with regard to the parasympathetic system (Fig. 7).

Figs 7(a) and (b) show that the delay due to the sympathetic as well as the parasympathetic system was not changing as the fatigue increased, while

(a)

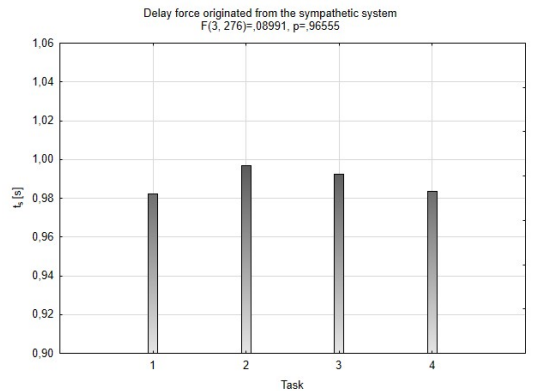

(c)

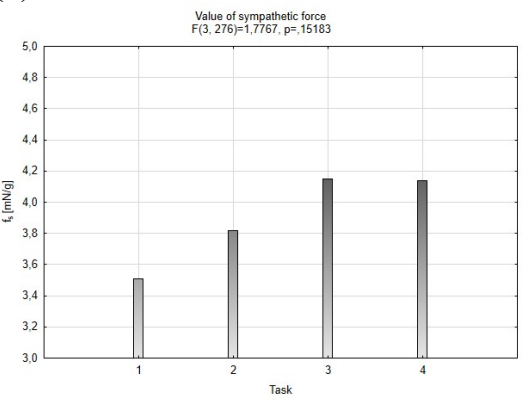

(b)

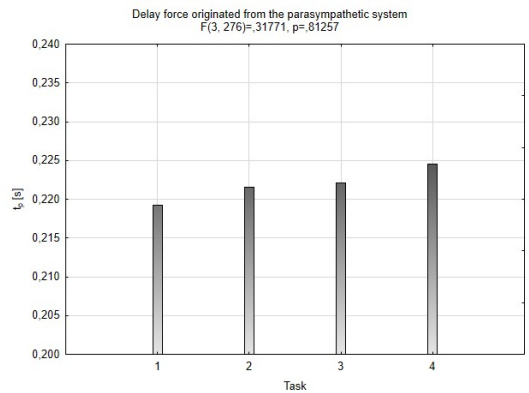

(d)

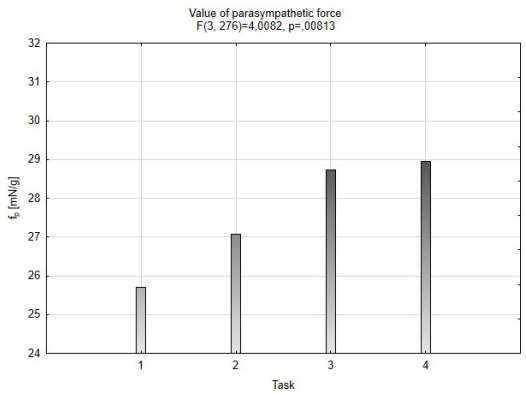

Figure 7: Changes in the parameters: (a) $t_{s}$, (b) $t_{p}$, (c) $f_{s}$, (d) $f_{p}$ at sequential measurements in the experimental procedure. 
the opposite was true for parameters $f_{s}, f_{p}$ presented in Figs 7(c) and (d). The effect of the parasympathetic systems was significant and evident already at the second measurement (about 1:30 am). A somewhat less pronounced effect was observed for the sympathetic nervous system.

(a)

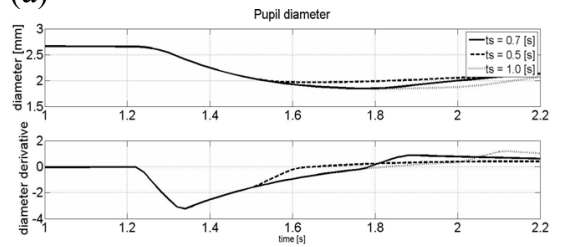

(c)

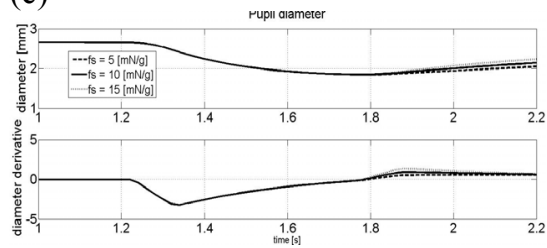

(b)

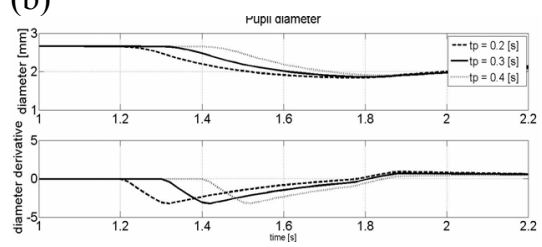

(d)

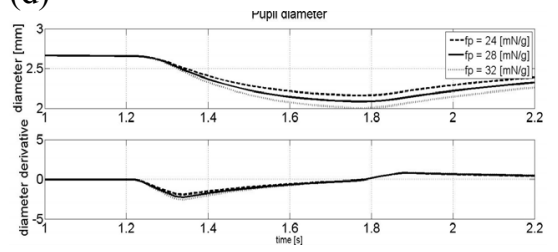

Figure 8: Dynamic change in the size of the pupil as a function of: (a) $t_{s}$, (b) $t_{p}$, (c) $f_{s}$ and (d) $f_{p}$.

The analysis was carried out using the STATISTICA data analysis software system, version 10 (StatSoft, Inc., 2011). Statistical analysis was performed using classic statistical methods based on the variance analysis.

\section{Discussion}

The implementation of Kelvin-Voight's model for the purposes of PLR characterization will allow for highly accurate assessment of the changes in pupillary behavior throughout the duration of the pupillary light reflex. Implementation of the system facilitated elimination of the basic drawbacks and limitations of the classic method of PLR assessment. These are due to the fact that the basic indicator values or range limits are determined at specific time points of the reflex. Due to the high speed of changes in the pupil size, particularly during the first phase of the reflex, shifting the detection time point by one hundredth of second may lead to errors of as much as over $10 \%$. This is unacceptable when the PLR and the assessment module are to be used, in line with the authors' assumptions, as a detector of early signs of fatigue. Only slight variations in the pupillary reflex records are observed in such cases.

Sometimes, the reflex signal may be obscured by the subject shutting their eyelid involuntarily. Thus, the measurement using the classic method of PLR assessment becomes useless. Depending on the moment of the disturbance, one should either repeat the entire measurement or significantly reduce the number of parameters that may be determined from such records. Another problem consists of the accuracy of the measurement (detection) of the pupil size. In case of a low 
resolution of the measurement system, the recorded signal does not fully reflect the full dynamics of PLR and the assessment becomes inaccurate. Too accurate measurement of pupil size may also be of a problem. In such case, the assessment may be disturbed by the presence of the oscillation wave, i.e. involuntary, oscillating movements of pupils that might modulate the PLR.

As shown by the results presented in the charts in Figs 7 and $8(d)$ and the results described in [15], it is possible to assess the level of fatigue while the subject performs the operator tasks. The currently used method for determination of PUI requires specific experimental conditions (full blackout) and is time-consuming (lasting 11 minutes).

The use of a dynamic model with estimated parameters ensuring accurate tracing of the actual pupillary reflex provides a solution to these problems and ensures an accurate, reliable and fast method for estimating the fatigue level.

\section{Conclusions}

Owing to proper implementation of the model, we managed to attain a high level of its adaptation to real data ( $>90 \%)$. Parameters assigned by the model, such as: elastic constant of the dilator and constrictor, static force pupil at rest, radius of the constrictor at rest, radius of the pupil at rest, delay force originated from the parasympathetic system, and delay force originated from the sympathetic system, were compared with a snapshot assigned by the AMTech system. The results show their strong correlation, and the model shows high sensitivity to changes in the shape of the reflex curve, caused by sleep deprivation introduced in the experiment.

The presented in the article dynamic model of pupillary reflex evaluation is a component of the system of fatigue evaluation developed by the authors [21]. In this solution, the measurements are mostly conducted with the use of an optical head with two side cameras with adjustable location, observing the eyes, with illuminators and infrared filters. The head was mounted in a goggle-type construction.

\section{Acknowledgement}

The work was co-financed by the National Centre for Research and Development, project no. DOBR/0038/R/ID2/2013/03.

\section{References}

[1] Różanowski K, Murawski K. Optical sensor to monitor pupillary light reflex. Acta Physica Polonica A. 2013; 124(3): 558-62.

[2] Różanowski K, Murawski K. An infrared sensor for eye tracking in a harsh car environment. Acta Physica Polonica A. 2012; 122(5): 874-79. 
[3] Tilmant C, Charavel M, Ponrouch M, Gindre G, Sarry L, Boire JY. Monitoring and modeling of pupillary dynamics: Study of the autonomous nervous system. Blaise Pascal University, France, OICMS. 2005; 441-49.

[4] Wilhelm B, Giedke H, Lüdtke H, Bittner E, Hofmann A, Wilhelm H. Daytime variations in central nervous system activation measured by a pupillographic sleepiness test. J Sleep Res. 2001 Mar; 10(1): 1-7, http://dx.doi.org/10.1046/j.1365-2869.2001.00239.x.

[5] Loewenfeld I, Lowenstein O. The pupil: Anatomy, physiology, and clinical applications, Iowa State University Press; 1993.

[6] Muppidi S, Scribner M, Gibbons CH, Adams-Huet B, Spaeth EB, Vernino S. A unique manifestation of pupillary fatigue in autoimmune autonomic ganglionopathy. Arch Neurol. 2012 May; 69(5): 644-48, http://dx.doi.org/ 10.1001/archneurol.2011.2143.

[7] Ferrari GL, Marques JLB, Gandhi R, Heller SR, Schneider FK, Tesfaye S, et al. Using dynamic pupillometry as a simple screening tool to detect autonomic aeuropathy in patients with diabetes: A pilot study. Biomed Eng Online. 2010; 9: 26, http://dx.doi.org/10.1186/1475-925X-9-26.

[8] Fotiou DF, Stergiou V, Tsiptsios D, Lithari C, Nakou M, Karlovasitou A. Cholinergic deficiency in Alzheimer's and Parkinson's disease: Evaluation with pupillometry, Int J Psychophysiol. 2009 Aug; 73(2): 143-49, http://dx.doi.org/10.1016/j.ijpsycho.2009.01.011.

[9] Höfle M, Kenntner-Mabiala R, Pauli P, Alpers GW. You can see pain in the eye: Pupillometry as an index of pain intensity under different luminance conditions. Original Research Article. Int J Psychophysiol. 2008 Dec; 70(3): 171-75.

[10] Payen JF, Isnardon S, Lavolaine J, Bouzat P, Vinclair M, Francony G. Pupillometry in anesthesia and critical care, Ann Fr Anesth Reanim. 2012; 31(6): e155-59.

[11] Kaltsatou A., Kouidi E., Lithari C., Koutlianos N., Fotiou F., Deligiannis A., Assessment of cardiac autonomic function in dynamic athletes by pupillometry, International Journal of Psychophysiology, Volume 77, Issue 3, September 2010: 283-84.

[12] Wilhelm BJ, Widmann A, Durst W, Heine C, Otto G. Objective and quantitative analysis of daytime sleepiness in physicians after night duties. Int J Psychophysiol. 2009 Jun; 72(3): 307-13.

[13] de Souza JK, Pinto MA, Vieira PG, Baron J, Tierra-Criollo CJ. An opensource, FireWire camera-based, Labview-controlled image acquisition system for automated, dynamic pupillometry and blink detection, Comput. Methods Programs Biomed. 2013 Dec; 112(3): 607-23.

[14] Canver MC, Cancer AC, Revere KE, Amado D, Bennet J, Chung DS. Novel mathematical algorithm for pupillometric data analysis, Computer Methods and Programs Biomedicine, Jan; 113 (1): 221-25.

[15] Różanowski K, Bernat M, Kamińska A. Estimation of operators' fatigue using optical methods for determination of pupil activity. International Journal of Occupational Medicine and Environmental Health. 2015; 28(2): $1-19$. 
[16] Yamaji K, Yoshitomi T, Usui S, Ohnishi Y. Mechanical properties of the rabbit iris smooth muscles. Vis. Res., vol. 43, 479-87, 2003.

[17] Usui S, and Hirata Y. Estimation of autonomic nervous activity using the inverse dynamic model of the pupil muscle plant, Ann. Biomed. Eng., vol. 23, 375-87, 1995.

[18] Xiaofei Fan, Gang Yao, Transient Pupillary Light Reflex Induced by a Short Light Flash, IEEE Transactions on Biomedical Engineering, vol. 58, no. 1, January 2011.

[19] Czarkowski M, Różanowski K. Polish Research Ethics Committees in the European Union system of assessing medical experiments, Science and engineering ethics. 2009, vol. 15: 201-12.

[20] Murawski K, Różanowski K, Krej M. Research and Parameter Optimization of the Pattern Recognition Algorithm for the Eye Tracking Infrared Sensor. Acta Physica Polonica A. Vol. 124 (2013) No. 3: 513-16.

[21] Murawski K, Różanowski K. Pattern Recognition Algorithm for Eye Tracker Sensor Video Data Analysis. Acta Physica Polonica A. Vol. 124 (2013) No. 3: 509-12.

[22] Urschitz MS, Heine K, Mendler L, Peters T, Wilhelm B, Poets CF. Pilot study on the validity of the pupillographic sleepiness test in children and adolescents. Sleep Medicine. 2014; 15(6): 720-23. 